African Journal of Drug \& Alcohol Studies, 8(1), 2009

Copyright (C) 2009, CRISA Publications

\title{
TO WHAT EXTENT DO YOUTH-FOCUSED PREVENTION PROGRAMMES \\ REFLECT EVIDENCE-BASED PRACTICES? FINDINGS FROM AN AUDIT OF ALCOHOL AND OTHER DRUG PREVENTION PROGRAMMES IN CAPE TOWN, SOUTH AFRICA
}

\author{
Nadine Burnhams*1 Bronwyn Myers ${ }^{2}$ Charles Parry ${ }^{3}$ \\ ${ }^{1}$ Alcohol and Drug Research Unit, Medical Research Council, Cape Town, South Africa \\ ${ }^{2}$ Alcohol and Drug Research Unit, Medical Research Council, Cape Town \\ ${ }^{3}$ Alcohol and Drug Research Unit, Medical Research Council, Cape Town and \\ Department of Psychiatry, Stellenbosch University
}

\begin{abstract}
Despite considerable effort directed towards youth focused prevention activities in South Africa, little is known about the content of these activities. A major concern is the extent to which substance abuse prevention programmes reflect evidence-based practices (EBPs). This paper reports on the findings from a cross-sectional audit of youth-focused alcohol and other drug prevention programmes conducted in the Cape Town Metropole in 2007. This audit explored the extent to which EBPs are used in prevention programmes and examined possible barriers to their adoption. Key findings suggest that most prevention programmes are not guided by EBP and are implemented in the absence of evidence on their effectiveness. The lack of a national regulatory regime for prevention programmes and limited funding are major barriers to implementing sustainable and effective prevention programmes. Based on these findings, strategies to assist in improving substance abuse prevention services and recommendations for improving service delivery systems are provided.
\end{abstract}

KeY WorDS: Youth, prevention programmes, South Africa, evidence-based practices

\section{INTRODUCTION}

South Africa has experienced escalating levels of alcohol and other drug (AOD) use during its transition from apartheid to democracy, particularly among young people of school-going age. For example, school surveys found that more than a third of male high school students in Cape Town and over half of male students in Durban reported binge drinking episodes in 1997 and 1998 respectively (Parry et al., 2004a). Heavy drinking by school-goers is significantly associated with absenteeism, academic failure, risky sexual behaviour (Flisher et al., 2003), increased risk for sexual victimisation, and increased likelihood of other drug use (Brooke et al., 2006; Grossman et al., 1998; King et al., 2003;). These findings, together with findings

Corresponding author: Nadine Burnhams*, Alcohol and Drug Research Unit, Medical Research Council, PO Box 19070, Tygerberg, 7505 Email: nadine.harker.burnhams@mrc.ac.za 
from the 2002 Youth Risk Behaviour Survey (Reddy et al., 2003) which point to high prevalence rates for binge drinking and lifetime cannabis use, suggest that a significant proportion of South African adolescents are at risk for developing AOD problems (Parry, 2005). This highlights the need for effective prevention interventions that target South African youth. AOD use increases during adolescence making it a critical time for AOD service providers to engage in prevention and brief intervention initiatives to prevent the initiation of, and limit the negative consequences associated with AOD use (Flisher et al., 2003).

These high levels of AOD use raise questions about the success of efforts to prevent or delay substance use among young people in South Africa. Various concerns have been raised about the quality and effectiveness of prevention services in South Africa. These concerns are partly due to the fact that there is no legislation that regulates and oversees the training, qualifications, and competencies of prevention service providers as there are for in and out patient substance abuse treatment (National Department of Social Development, 2008). In addition, there are no minimum norms and standards to guide prevention interventions. This lack of an organisational framework through which prevention services can effectively and efficiently be delivered impacts not only on service quality, but also on the diffusion of new ideas and technologies which are pertinent to prevention science (Merrill et al., 2006). Despite this, there has been little research to date on the content and quality of AOD prevention services in the country and most organisations render prevention services without knowledge of their effectiveness.

One indication that AOD prevention services are of an adequate quality is the extent to which they adopt evidence-based practices (EBPs). EBPs are practices, interventions or programmes for which there is a large body of research evidence in support of its effectiveness (Myers et al., 2008). These EBPs reach beyond geographical boundaries and consequently can be applied to the South African context with only minor adjustments. According to the National Institute on Drug Abuse (NIDA) and others (Loxley et al., 2003; Foxcroft et al., 2003, UNODC, 2004), an important EBP for youth focused prevention is the enhancing of individual protective factors and the minimization of risk factors. Another EBP is encouraging and equipping individuals with life skills, including drug resistance skills. Encouraging family participation and maintaining a focus on the family within programmes is also a key EBP, as is the use of culturally sensitive and appropriate methods. Cultural sensitivity can be defined as "the extent to which ethnic/ cultural characteristics, experiences, norms, values, behavioural patterns, and beliefs of a target population as well as relevant historical, environmental and social forces are incorporated in the design, delivery and evaluation of targeted health promotion materials and programmes" (Resnicow et al., 2000). The use of culturally sensitive material in prevention programmes is absolutely vital, considering the diversity of the population in Cape Town. According to Census 2001 data, $27 \%$ of persons residing in the Western Cape are Black/African, $1 \%$ are Indian, $18 \%$ White and $54 \%$ fit the Coloured race profile (STATSSA, 2001). Other EBPs include the use of interactive teaching methods instead of traditional didactic teaching methods and the use of programmes that are cost effective and easy to implement.

\section{AIMS}

The prevention audit, conducted as a pilot study, was a direct response to the need for additional information on the quality of AOD prevention programmes in South Africa. The paper below sought to explore the extent to which EBPs are used in AOD prevention programmes that target young people under the age of 21 years in Cape Town, South Africa. Possible barriers to adopting EBPs are also explored.

\section{METHODS}

A cross-sectional survey of AOD prevention programmes in the Cape Town metropole 
was conducted in 2007. The sample comprised all Directors of organizations in the greater Cape Town metropole providing prevention services, either as a primary or secondary focus. The sample frame was initially constructed from the Medical Research Council of South Africa's list of known prevention services. Key informant interviews were also conducted to assist in identifying other service providers that were not part of this list. The final list comprised 47 directors. Organisations were contacted via telephone and asked to participate in the study. This initial contact was followed by a letter confirming participation in the study and a questionnaire pack. The initial response rate was poor and the date for submission was extended three times to ensure a response rate of at least $65 \%$. The final response rate was $74 \%(\mathrm{n}=35)$.

Data for the audit were collected by means of a 10-page open-ended, structured questionnaire comprising 44 questions all in English. The questionnaire took on average thirty minutes to complete. The questionnaire included questions on the characteristics of the organisation and its programmes, the types of prevention programmes offered, staffing characteristics, and monitoring and evaluation methods and allows for assessing whether individual programmes follow EBPs. Statistics for this study were computed using the Statistical Package for the Social Sciences (Norusis/ SPSS Inc., 2007). The focus was on descriptive data and no hypothesis testing took place.

\section{RESULTS}

\section{Profile of AOD prevention programmes in Cape Town}

Prevention programmes in Cape Town are most likely to take the form of educational programmes (91\%) which aim to raise awareness of AODs by providing knowledge about AODs and their consequences. Only 57\% of organisations offer skills-based prevention programmes and just over half (51\%) offer both education and life skills programmes depending on the request from the consumer. A small proportion of organisations $(6 \%)$ continue to rely on the sharing of personal experiences of drug use as a means of preventing or deterring AOD use.

Most programmes are once-off interventions occurring on an ad hoc basis and $40 \%$ of organisations do not conduct long-term repeated interventions. More specifically, more than two-thirds $(69 \%)$ of organisations working in school settings only offer short-term, once-off awareness-raising activities and interventions. On average, the length of each prevention session ranges from 45 minutes (for once-off education sessions) to 3 hours (life skills sessions).

\section{The use of culturally sensitive methods}

The audit also explored the extent to which programmes were sensitive to diverse cultures (see Figure 2). Findings yielded some inconsistencies. While most organisations reported using methods that were culturally sensitive, respondents were unable to provide examples of the culturally sensitive methods that they employed; raising doubts about the validity of the findings. For instance, nine out of ten organisations reported using age-appropriate content material, but only one organisation provided an example of such material.

\section{Risk and protective factors}

For $85 \%$ of respondents, the reduction of risk factors for the initiation of AOD use was a primary objective of their programme. Yet many risk factors remain poorly addressed by programmes (see Table 1). For instance, less than two-thirds of programmes addressed poor parenting skills, only half targeted membership of deviant peer groups, and only a third targeted either learning difficulties and poor school performance or mental health problems.

In terms of protective factors, most respondents reported that their programmes enhanced parental involvement and positive peer relations and encouraged healthy social norms. In addition, roughly three-quarters of organisations reported their programmes developed psychosocial skills such as life skills, coping skills, decision making skills, and goal setting skills. In 


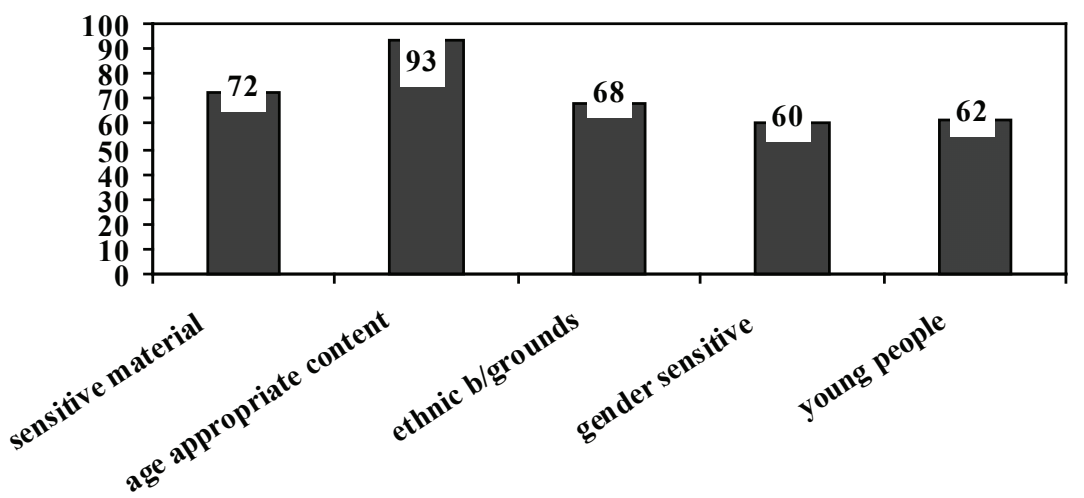

Figure 1: Proportion (\%) of organisations reporting the use of culturally sensitive methods within primary prevention services

Table 1. Proportion (\%) of organisations addressing specific risk factors

\begin{tabular}{ll}
\hline \multicolumn{1}{c}{$\begin{array}{c}\text { Risk factors addressed in } \\
\text { prevention programmes }\end{array}$} & \% \\
\hline $\begin{array}{l}\text { Substance abuse among adult role } \\
\text { models }\end{array}$ & 71 \\
Poor parenting skills & 63 \\
Poor family relations and bonding & 77 \\
Membership to deviant peer groups & 54 \\
(gangs, etc.) & \\
Learning difficulties/poor school & 37 \\
performance & \\
Peer pressure & 80 \\
Mental health problems & 37 \\
\hline
\end{tabular}

contrast, a much smaller proportion encouraged parental monitoring $(57 \%)$, involvement in prosocial institutions $(41 \%)$ or conducted health promotion activities (39\%) (Table 2).

\section{Barriers to adopting EBPS}

Less than half $(48 \%)$ of the organisations reported allocating money to prevention activities. Of these, a quarter (22\%) indicated that $50 \%$ or less of their annual budget was allocated to prevention activities; $14 \%$ indicated an allocation amount of between $50-95 \%$ and only $11 \%$ reported that their entire budget was spent on prevention services. Twenty-three percent indicated that no budget was allocated for prevention services and $29 \%$ of organisations did not answer any budget related questions. Fifty- one percent of organisations indicated that substance abuse prevention is a secondary rather than a primary focus, because of poor funding opportunities for this kind of intervention.

Gaps in training are a particular barrier to the adoption of EBPs. Fifty-one percent of all respondents had no specialised training in the prevention of risky behaviours, $29 \%$ had no training in AOD use disorders, and roughly a third had no training in working with young people. In addition, only $62 \%$ of organizations provide supervision to their prevention workers either on a daily $(9 \%)$, weekly $(35 \%)$ or monthly (18\%) basis.

Table 2: Proportion (\%) of organisations enhancing specific protective factors

\begin{tabular}{lc}
\hline \multicolumn{1}{c}{ Protective factors addressed } & \% \\
\hline Parental involvement & 83 \\
Parental monitoring & 57 \\
Adopting healthy social norms & 85 \\
Decision making skills & 77 \\
Conflict negotiation & 66 \\
Life skills & 74 \\
Coping & 74 \\
Goal setting & 77 \\
Self-efficacy & 77 \\
Positive peer relationships & 91 \\
Pro-social institutions e.g. Church; sports, & 41 \\
volunteering & \\
Health promotion & 39 \\
\hline
\end{tabular}




\section{DISCUSSION}

Findings from this study suggest that EBPs have not been widely adopted by AOD prevention programmes targeting young people in Cape Town and are contrary to the broad principles of effective prevention. Although EBP guidelines note that once off campaigns are largely ineffective (Loxley et al., 2003), prevention programmes in Cape Town tend to be once-off activities that target a large quantity of people. Few programmes have multiple years of intervention. A possible reason for this may lie in the fact that funders are often more interested in the large quantity of people reached than the quality or real impact of the programmes and it is easier to reach large audiences with once-off programmes. Evidence of large numbers reached is often used to influence funders when applying for programme funding.

Another EBP is that AOD programmes not only focus on information-sharing and awareness raising activities but are also skills-based (Mentor UK, 2006). In South Africa, schoolbased AOD prevention programmes are very popular as schools facilitate easy access to a large part of the young population. In addition to this, schools often assume responsibility for addressing a variety of social and health problems (Sloboda \& Bukoski, 2003). Contrary to EBP guidelines (NIDA, 2003), this audit found that the majority of organisations working in school settings offer once-off awareness and education sessions to schools rather than skills based programmes. These sessions aim to prevent young people from misusing substances by providing knowledge about substances and their consequences. Within this approach, the overall premise is that individuals will make more rational decisions about AOD use if they understand the risks associated with AOD use (Foxcroft et al., 2003). The danger with this approach is that the information provided may stimulate curiosity and potentially increase AOD use by young people (Sloboda \& Bokoski, 2003). Apart from this, there is a large body of evidence to suggest that information provision alone does not prevent or change be- haviours (Komro et al., 2008; Medina-Mora, 2005). It is therefore essential that AOD prevention programmes not only provide information but also build skills to empower individuals to make healthy choices and avoid the use of AODs (Foxcroft et al., 2003). These skills could include communication and negotiation, social problem-solving and decision-making, conflict resolution, and specific drug refusal skills (Atkinson, 2004).

Related to this, reducing risk factors for and increasing protective factors against the initiation of AOD use not only influences developmental pathways but is an important evidencebased approach to prevention (Loxley et al., 2003; NIDA, 2003; UNODC, 2002). Our findings suggest that many programmes do attempt to meet this practice guideline. However, the extent to which they are able to do so effectively is questionable as reducing risk factors and enhancing protective factors requires sustained behavioural interventions rather than once-off prevention campaigns that educate audiences about risk factors. In addition, few AOD programmes in Cape Town address membership of deviant peer groups, learning difficulties and poor school performance or mental health problems. This is worrisome as there is a high prevalence of gang-related activities in Cape Town's poorer communities (Wilkinson, 2000) with AOD use, particularly methamphetamine use, playing a key role in the gang culture within these communities (Kapp, 2008). In addition, the Western Cape Education Department has seen high school dropout rates as well as an increase in the incidence of depression among young people (Townsend et al., 2004). Various studies have found associations between tobacco use (and other risk behaviours), substance use and school dropout (Flisher et al., 2003) and associations between methamphetamine use and poor mental health functioning, aggression and depression (Plüddemann et al. (in press). Although the reasons for these associations may not always be clear, they do point to the importance of jointly addressing these risk factors, especially as risk factors act in a cumulative way over time (Loxley et al., 2003). 
Another EBP is the use of culturally-sensitive AOD prevention programmes as no single prevention programme is appropriate to all individuals, target groups or communities. More specifically, prevention services should be adapted to meet the developmental needs of children in specific age groups, be gender-sensitive, and culturally appropriate (that is linguistically appropriate, contextually correct, and sensitive to cultural diversity) (Myers et al., 2008). Attempts to impose values, practices or judgments of one group on another without considering the culture of and resources available to the target audience have been shown to be destined for failure (Gullotta \& Bloom, 2003). Our findings raise concerns about whether programmes in Cape Town are sufficiently sensitive to gender and cultural differences. More specifically, findings suggest that a significant proportion of programmes are not gender sensitive, are not adapted to meet the needs of young people and do not employ ethnically diverse facilitators. These findings are worrisome given the city's cultural diversity.

Findings also highlight two barriers to implementing EBPs. The first is limited funding and financial resources. Organisations (particularly non-profit organisations) are under pressure to scale up the coverage of their prevention services and yet are limited by a lack of financial resources. In order to resolve this dilemma, organisations often render services on an ad hoc basis which incurs minimal costs to the service provider but is contrary to EBP guidelines regarding multiple years of intervention (Komro et al., 2008; Medina-Mora, 2005). While this approach does not lead to sustained behaviour change, it allows organisations to address community needs and is cheaper than repeat interventions. To address this barrier, additional funding should be injected into prevention activities. Funds allocated specifically for prevention work would encourage organisations to adapt and expand existing programmes to fit the needs of diverse age groups, enable them to offer programmes that are sustainable, and allow them to reach all levels of the community.
Funding, however, should be contingent on organisations following EBP for prevention and targeting those most at risk for developing AOD problems.

The second barrier is the limited regulation of AOD prevention programmes and prevention practitioners. South Africa currently does not have an effective framework guiding prevention services. Specifically, the complexity of AOD problems, its impact on public health, advances in prevention research as well as an escalating demand for services suggests that the manner in which prevention initiatives are conducted should be based on the best possible evidence (CARBC, 2006). Implementing EBPs requires AOD prevention workers to be adequately trained in AOD issues, in EBPs, and also in prevention science (Atkinson et al., 2004). Prevention workers should be kept abreast of new research and programme developments within the substance abuse field and other related fields. In addition, regular and ongoing supervision of prevention workers offers the opportunity of identifying the learning needs of prevention workers, monitoring outputs and ensuring the delivery of a quality service. Yet our findings highlight gaps in training and low limited supervision of prevention workers, suggesting organizations provide workers with the necessary training and supervision, especially given the scientific complexity of these services. In instances where supervision is provided, information on the quality and quantity of supervision remains unclear. These findings, together with the lack of minimum norms and standards for effective practice and the accreditation of individual prevention workers and the prevention services, highlights the need to establish a regulatory regime for prevention services. Such an organisational framework should help facilitate improvements in service effectiveness and quality.

Although the audit was successful in gathering information on prevention programmes in Cape Town, the extent to which findings can be generalized to other settings in South Africa is questionable. This highlights the 
need for a national audit of prevention services. In addition, the modest response rate raises some questions about some of the findings. For instance, even though organisations did indicate the use of culturally sensitive methods and conducting regular outcome and process evaluations, discrepancies such as an inability to provide examples of culturally sensitive methods and failing to provide a description of evaluation designs and associated findings were noted. These discrepancies could be attributable to the poor understanding of terminology used in the questionnaire suggesting that the study would have benefited from a qualitative component and face-to-face interviews. Such a component would have provided insight into the inner workings of the programmes and would have afforded the investigators the opportunity to follow-up on questions that required clarification. Lastly, a focus on prevention activities involving environmental determinants of AOD use would have been advantageous to the study. The inclusion of such a component would have provided a rich description of prevention at the individual, collective and environmental level in Cape Town.

\section{CONCLUSION}

Findings from this study address an important gap in our knowledge of AOD service provision in Cape Town. This study revealed that EBPs are not widely adopted by prevention programmes aimed at preventing AOD abuse among young people. More specifically, the prevention programmes available often lack continuity and are presented in the absence of evidence on their effectiveness. Lack of funding for AOD prevention is a major limitation for the adoption of EBPs as is the lack of appropriately trained and regulated service providers. Implementing programmes that meet best practice standards require a steady flow of income and trained prevention workers. Without this income, it is difficult to implement sustainable programmes and retain qualified and skilled personnel.

\section{REFERENCES}

Atkinson, A.J, Tolbert, I.K. (2004) Our common language: a quick Guide to prevention terminology in Virginia. GOSAP. Virginia

Brook JS, Morojele NK, Pahl T \& Brook DW. (2006). Predictors of drug use among South African adolescents. Journal of Adolescent Health, 38 (1) 26-34.

Ennett, S.T., Reingwalt, C.L., Thorne, J., Rohrbach, L.A., Viscus, A., Simons-Rudolph, A. \& Jones, S. (2003). A comparison of current practice in school-based substance use prevention programmes with Metaanalysis findings. Prevention Science, 4 (1) $1-13$.

Flisher A.J., Parry, C.D.H., Evans, J., Muller, M., \& Lombard, C. (2003) Substance Use by Adolescents in Cape Town: Prevalence and Correlates Journal of Adolescent Health 32,58-65

Foxcroft, D.R., Ireland, D., Lister-Sharp, G. \& Breen, R. (2003). Longer-term primary prevention for alcohol misuse in young people: a systematic review. Addiction, 1 , (93) 397-411.

Grossman, M., Chaloupka, F.J., \& Sirtalan, J. (1998). An empirical analysis of alcohol addiction: Results from the Monitoring the Future panels. Economic Inquiry 36, 49-48.

Hallfours, D. \& Van Dorn, R.A. (2002) Strengthening the role of two key institutions in the prevention of adolescent substance abuse. Journal of Adolescent Health, 30 (1) 17-28.

Kapp, C. (2008) Crystal meth boom adds to South Africa's health challenges. Lancet. 371 (9608) 193-194.

King, G., Flisher, A.J., Noubary, F., Reece, R., Marais, A. \& Lombard, C. (2004) Substance abuse and behavioural correlates of sexual assault among South African adolescents. Child Abuse \& Neglect 28, 683-696.

Komro, K.A., Perry, C.L, Veblen-Mortensen, S., Farbakhsh, K., Toomey, T.L., Stigler, M.H., Jones-Webb, R., Kugler, K.C., 
Pasch, K.E., \& Williams, C.L., (2008). Outcomes from a randomized controlled trial of a multi-component alcohol use preventive intervention for urban youth: Project Northland Chicago. Addiction 103, 606-618.

Loxley, W., Toumbourou, J. \& Stockwell, T. (2003). The prevention of substance use, risk and harm in Australia: a review of the evidence. The National Drug Research Institute and the Centre for Adolescent Health. Australia

McBride, N. (2003). Systematic literature review of school drug education. Manuscript presented at the Freemantle conference.

Medina-Mora, ME. (2005). Prevention of substance abuse: a brief overview. World Psychiatry. 4 (1), 25-30.

Mentor UK. 2005. Principles of effective practice: Mentor Foundation's scientific advisory network. London: Mentor.

Merrill, J.C., Pinsky, I., Killeya-Jones, L.A., Sloboda, Z. \& Dilascio,T. (2006) Substance Abuse prevention infrastructure: a survey-based study of the organisational structure and function of the D.A.R.E. program. Substance Abuse Treatment Prevention Policy. 6 (1) 1-25.

Myers, B., Harker, N., Fakier, N., Kader, R. \& Mazok, C. (2008) A review of evidencebased interventions for the prevention and treatment of substance use disorders. Technical Report. Medical Research Council. South Africa

National Department of Social Development (2008) Minimum norms and standards for out-patient treatment centres. A manual developed with the support of the United Nations (office on drugs and crime). Pretoria: South Africa.

National Institutes of Health (2003) National Institute on Drug Abuse: Preventing drug use among children and adolescents. Bethesda: NIH.

Parry, C.D.H. (2005) South Africa: Alcohol today. Society for the study of Addiction. Addiction, 100, 426-429.

Parry, C.D.H., Myers, B., Morojele, N.K., Flisher, A.J., Bhana, A., Donson, H. \&
Plüddemann, A. (2004) Trends in adolescent alcohol and other drug use: findings from three sentinel sites in South Africa (1997-2001) Journal of Adolescence. 27, 429-440.

Plüddemann, A., Flisher, A.J., Mathews, C., Carney, T., \& Lombard, C. (2008). Adolescent methamphetamine use and sexual risk behaviour in secondary school students in Cape Town, South Africa. Drug \& Alcohol Review, 27, 687-692.

Reddy, S.P., Panday, S., Swart, D., Jinabhai, C.C., Amosun, S.L., James, S., Monyeki, K.D., Stevens, G., \& Morojele, N. (2003). Umthente Uhlaba Usamila: The First SA National Youth Risk Behaviour Survey, 2002. Cape Town: South African Medical Research Council.

Resnicow, K., Braithwaite, R.L., Ahluwalia, J.S., \& Butler, J. (2000). Cultural sensitivity in substance use prevention. Journal of Community Psychology. 28, (3), 271-290.

Sloboda, Z. \& Bukoski, W.J. 2003. Handbook of drug abuse prevention: Theory, Science, and Practice. New YorK: Kluwer Academic/Plenum publishers.

Statistics South Africa (STATSSA). (2001) Census 2001. www.statssa.co.za

The Centre for Addictions Research of British Columbia.(CARBC). (2006). Following the evidence: preventing harms from substnace use in $B C$. Library and Archives Canada Cataloguing in Publication Data. British Columbia.

Townsend, L., Flisher, A.J., Chikobvu, P., Lombard, C. \& King G. Relationship between substance use and high school dropout in Cape Town, South Africa. 132nd Annual Meeting of the American Public Health Association, Washington, DC, U.S.A., 6 - 10 November 2004.

United Nations Office on Drugs and Crime. (2004). Conducting Effective Substance Abuse Prevention Work among the Youth in South Africa: Guidelines. Pretoria: UNODC.

Wilkinson, P. (2000) City Profile, Cape Town. Cities 17 (3), 195-205. 